\title{
HIV/AIDS Dynamics with Three Control Strategies: The Role of Incidence Function
}

\author{
Emmanuelina L. Kateme, ${ }^{1}$ Jean M. Tchuenche, ${ }^{1}$ \\ and Senelani D. Hove-Musekwa ${ }^{2}$ \\ ${ }^{1}$ Mathematics Department, University of Dar es Salaam, Dar es Salaam, Tanzania \\ ${ }^{2}$ Department of Applied Mathematics, National University of Science and Technology, \\ Bulawayo, Zimbabwe \\ Correspondence should be addressed to Emmanuelina L. Kateme, emkateme@gmail.com
}

Received 3 March 2012; Accepted 29 April 2012

Academic Editors: H. Akçay, C. Lu, and G. Psihoyios

Copyright @ 2012 Emmanuelina L. Kateme et al. This is an open access article distributed under the Creative Commons Attribution License, which permits unrestricted use, distribution, and reproduction in any medium, provided the original work is properly cited.

The type of incidence function used in epidemiological models is generally a matter of choice and convenience. Basic deterministic HIV/AIDS models with standard and saturated incidences are developed and extended to include three control measures, namely, public health educational campaigns, condom use, and treatment. The potential impact of these incidence functions on long-term projection of the disease dynamics is theoretically assessed, both qualitatively and quantitatively. Conditions for the stability of the model steady states are provided. The model with saturated incidence yields a higher number of secondary infections compared to the same outcome in the standard incidence formulation.

\section{Introduction}

HIV / AIDS prevention and control is a public health priority in light of the global pandemic and the elevated death toll (27 million since the disease was identified in 1981). To assess the impact of incidence functions in the estimation of the long-term dynamics of the disease, mathematical models of infectious diseases are useful tools for comparing control strategies and identifying key disease drivers as well as important areas of uncertainty that may be prioritized for urgent research. Large amount of work done on modeling the spread of HIV has been largely restricted to ordinary differential equations, though studies which have incorporated the combination of condom use, public health education campaigns, and treatment of infected individuals for eradication of the epidemic in the saturation incidence are uncommon (see [1-4] and the references therein). 
Standard incidence models with constant total population are essentially mass action models [5], but reality is somewhere in between these two popular formulations. They appear to be the most widely used in the mathematical epidemiology repertoire, and some studies have suggested that the standard incidence formulation is more realistic for human diseases. To model the inhibition effect resulting from behavioral change or the crowding effect of infected individuals, neither mass action nor standard incidence functions is adequate. This is better captured using a saturation incidence formulation. The role of saturation and standard incidence functions on the dynamics of HIV is assessed with and without control measures.

Although the choice of one formulation over the other really depends on the disease being modeled and more often on the need for analytical tractability, it is imperative to assess the impact of this choice on initial disease threshold. We are not aware of any study that has investigated this impact, and it is our hope that this study will shed some light on whether using one formulation over the other over or underestimate the burden of the disease.

The rest of the paper is organized as follows: the model framework is presented in Section 2, followed by the basic model and its analysis in Section 3. The model with control measures is formulated and analyzed it Section 4, with graphical representations provided for illustration. Section 5 concludes the paper.

\section{Model Framework}

The proposed deterministic HIV/AIDS model subdivides the total population at time $t$, denoted by $N(t)$, into susceptibles or HIV negatives, $S(t)$, HIV positives or infectives who do not know their status, $I_{1}(t)$, HIV positives or infectives aware of their status, $I_{2}(t)$, pre-AIDS population, $P(t)$, and full blown AIDS individuals, $A(t)$. The total sexually active population size at time $t$ is thus given by $N(t)=S(t)+I_{1}(t)+I_{2}+P(t)+A(t)$. Some models have assumed that only a fraction of individuals in the AIDS class are sexually active but fail to give a clear estimate of this fraction $[4,6]$. However, the long-term dynamics of the disease is not affected by this small withdrawal, and, for this reason, such categorization is not considered herein.

Suppose that $\beta$ is the effective contact rate (i.e., the average number of contacts sufficient to transmit infection) per individual per unit time. Then $\beta I_{1} / N$ is the average number of contacts a susceptible individual makes with infective individuals per unit time. Therefore, the number of new infections coming from susceptibles is $\lambda S$ where $\lambda=\beta I_{1} / N$ represents the force of infection (this rate at which susceptible individuals contract the disease is measured by reference to the serological status of an individual through time with respect to antibodies specific to the infectious agent in question). When the effective contact rate $\beta$ is constant, the force of infection is referred to as a standard incidence (frequencydependent incidence), and $I / N$ represents the fraction of the infected population. When the total population size $N$ is quite large, since the number of contacts made by an infective per unit time should be limited or should grow less rapidly as the total population size $N$ increases, the constant contact rate, $\beta$, may be more realistic [7].

However, the contact rate is not always linear. As the number of infectives rises, the number of susceptibles usually declines making it more difficult for contacts to be made. This means that there is a saturation effect in the contact rate as the number of infectives increases. The dynamics of an epidemic to a large extent is determined by how new infections are generated and the population mixing pattern. Incidence functions determine the rise and fall of epidemics. The incidence rate of a disease is the rate at which new cases of infection 
arise in a population and play an important role in the study of mathematical epidemiology [7].

The population mixes homogeneously, which means that susceptible individuals are equally likely to be infected by an infectious individual. Homogeneity is equivalent to assuming that the conditional mean distribution of parameters of interest is invariant across population subgroups. Nonlinearities can be approximated by a variety of forms. Xiao and Ruan [8] considered a saturation incidence rate of the form $k I S /\left(1+\alpha I^{2}\right)$ where $k I$ measures the infection force of the disease and $1 /\left(1+\alpha I^{2}\right)$ describes the psychological or inhibitory effect from the behavioral change of the susceptible individuals when the number of infectives is very large $(k, \alpha>0)$. Liu et al. [9] focussed on the incidence rate $\lambda I^{p} S^{q}$ and noted that the global results obtained may be less generally applicable than the local results where $p$ and $q$ are positive parameters. Zhang and Ma [7] considered a saturation contact rate $C(N)=b N /(1+b N+\sqrt{1+2 b N})$ where $b$ is a nonnegative parameter. In what follows, we will restrict our attention to a standard incidence function of the form $\beta I / N$ and a saturation incidence function of the form $\beta I /(1+\alpha I)$ to investigate how the dynamics, in terms of the reproduction numbers changes with change in the incidence function. The force of infection for the standard incidence model is given by

$$
\lambda_{\mathrm{st}}=\frac{\beta\left(I_{1}+k_{1} I_{2}+k_{2} P+k_{3} A\right)}{N},
$$

where $\beta$ is the effective contact rate. The modification parameters $k_{1}<1, k_{3}>1$ and $k_{2}<k_{3}$ account for the relative infectivity of individuals in the $I_{2}, P$, and $A$ classes when compared to individuals in the $I_{1}$ class. This implies that individuals in the $A$ class are more infectious than those in the $I_{1}$ class due to their higher viral load. Likewise, individuals in the $I_{2}$ class tend to infect less compared to those in the $I_{1}$ class because of their status awareness (individuals in the $I_{2}$ class may choose to use preventive measures and change their behavior and thus may contribute little in spreading the infection). Individuals in the $P$ class are less infectious than those in the $A$ class because of their lower viral load but more infectious than those in the $I_{1}$ and $I_{2}$ classes. The modification parameters thus satisfy the following relation, $k_{1}<k_{2}<k_{3}$.

In the model with saturated incidence rate, $\lambda_{\text {sat }}$ (the force of infection at time $t$ ) is given by

$$
\lambda_{\text {sat }}=\frac{\beta\left(I_{1}+k_{1} I_{2}+k_{2} P+k_{3} A\right)}{1+\omega\left(I_{1}+I_{2}+P+A\right)}
$$

where $1 /\left(1+\omega\left(I_{1}+I_{2}+P+A\right)\right)$ measures the inhibition effect from behavioral change of susceptible individuals when their number increases or from the crowding effect of the infective individuals [8]. The nonnegative constant $\omega$ is the parameter that measures the extent of psychological or inhibitory effect (detrimental effect if $0<\omega<1$, beneficial or positive effect if $\omega>1$ ). The nonmonotonic functions in (2.1) and (2.2) capture the psychological effects of increasing infectives in the population [10]. For a very large number of infective individuals, the force of infection may decrease as this number increases due to the fact that in the presence of large number of infectives, the population may tend to reduce the number of contacts per unit time [8].

The sexually mature susceptible individuals are recruited into the population at a constant rate $\Lambda$. This subpopulation is reduced by infection, following effective contact with 
infected individuals at the rate $\lambda_{\text {st }}$ for the model with standard incidence rate. It is reduced further by natural death at a rate $\mu$ and emigration at a rate $\tau$. Thus, the rate of change of susceptible individuals with time is given by

$$
\frac{d S}{d t}=\Lambda-\lambda S-(\mu+\tau) S
$$

with $\lambda$ given by (2.1) and (2.2) for the standard incidence and the saturated incidence models, respectively.

Once an individual is infected, he/she becomes infectious and remain infectious (since treatment only suppresses the viral load). The population of HIV-positive individuals or infectives who do not know their status is increased by infection of susceptible individuals at the rate $\lambda$. The former is decreased by screening which leads to status awareness at the rate $\rho$, development of clinical symptoms, progression to full blown AIDS, and natural death at the rates $\theta, v$, and $\mu$, respectively. This population is further decreased by emigration at a rate $\tau$. Hence,

$$
\frac{d I_{1}}{d t}=\lambda S-\rho I_{1}-\theta I_{1}-v I_{1}-(\mu+\tau) I_{1}
$$

The population of HIV positives or infectives aware of their HIV status is generated by screening of unaware infectives at the rate $\rho$ and decreased by development of clinical symptoms at the rate $\sigma$, development of full blown AIDS at the rate $\eta$, natural death at the rate $\mu$, and emigration at the rate $\tau$, so that

$$
\frac{d I_{2}}{d t}=\rho I_{1}-\sigma I_{2}-\eta I_{2}-(\mu+\tau) I_{2}
$$

The pre-AIDS population is generated following development of clinical symptoms at the rate $\theta$ (for unaware infectives) and $\sigma$ (for aware infectives). The model assumes that there is no emigration of pre-AIDS individuals to other countries because they are symptomatic and physically weak compared to $I_{1}$ and $I_{2}$ individuals. This subpopulation is diminished by progression to full blown AIDS at the rate $\alpha$ and natural death at the rate $\mu$, so that

$$
\frac{d P}{d t}=\theta I_{1}+\sigma I_{2}-(\alpha+\mu) P
$$

Finally, the population of individuals with AIDS is increased by progression to full blown AIDS (at the rate $v$ for unaware infectives, $\eta$ for aware infectives, $\alpha$ for pre-AIDS individuals). It is decreased by natural death at the rate $\mu$ and by disease-induced mortality at the rate $\delta$. Thus,

$$
\frac{d A}{d t}=v I_{1}+\eta I_{2}+\alpha P-(\delta+\mu) A
$$




\section{The Basic Model}

Putting the above formulations and assumptions together give the following system of differential equations for the transmission dynamics of HIV/AIDS. The associated model variables and parameters are described in Tables 1 and 2, respectively:

$$
\begin{gathered}
\frac{d S}{d t}=\Lambda-\lambda S-(\mu+\tau) S, \\
\frac{d I_{1}}{d t}=\lambda S-\rho I_{1}-\theta I_{1}-v I_{1}-(\mu+\tau) I_{1} \\
\frac{d I_{2}}{d t}=\rho I_{1}-\sigma I_{2}-\eta I_{2}-(\mu+\tau) I_{2}, \\
\frac{d P}{d t}=\theta I_{1}+\sigma I_{2}-(\alpha+\mu) P, \\
\frac{d A}{d t}=v I_{1}+\eta I_{2}+\alpha P-(\delta+\mu) A .
\end{gathered}
$$

A schematic flow diagram of the model structure is depicted in Figure 1.

Since system (3.1) monitors a hypothetical human population, it is assumed that all the state variables and parameters are nonnegative for all $t \geq 0$. The model is well defined in $\Gamma=\left\{\left(S, I_{1}, I_{2}, P, A\right) \in \mathbb{R}_{+}^{5}: N(t) \leq \Lambda / \mu\right\}$, which is positively invariant and attracting [11].

\subsection{The Basic Model with Standard Incidence}

The basic model with standard incidence has a disease-free equilibrium given by

$$
E_{0}=\left(\frac{\Lambda}{\mu+\tau}, 0,0,0,0\right)
$$

Its reproduction number defined as the expected number of secondary infections generated by a single infective introduced into a naive/susceptible population in its entire period of infectiousness plays a vital role in the control and eradication of the disease. The threshold condition for the persistence or eradication of a disease determines whether an infection can invade and persist in a population [12]. Using the next generation operator [13],

$$
R_{0}^{\mathrm{st}}=\frac{\beta}{E_{1}}+\frac{\beta k_{1} \rho}{E_{1} B}+\frac{\beta k_{2}(\sigma \rho+\theta B)}{E_{1} B C}+\frac{\beta k_{3}[\alpha(\sigma \rho+\theta B)+C(\eta \rho+v B)]}{E_{1} B C D},
$$

where

$$
E_{1}=\rho+\theta+v+\mu+\tau, \quad B=\sigma+\eta+\mu+\tau, \quad C=\mu+\alpha, \quad D=\delta+\mu .
$$

Thus, from Theorem 2 in van den Driessche and Watmough [13], the following result holds. 
Table 1: State variables of the HIV / AIDS basic model.

\begin{tabular}{ll}
\hline Symbol & Description \\
\hline$S(t)$ & Susceptibles or HIV negatives at time $t$ \\
$I_{1}(t)$ & HIV-infected individuals unaware of their status \\
$I_{2}(t)$ & HIV-infected individuals aware of their status \\
$P(t)$ & Pre-AIDS individuals at time $t$ \\
$A(t)$ & AIDS individuals at time $t$ \\
\hline
\end{tabular}

Table 2: Parameters of the HIV/AIDS basic model.

\begin{tabular}{ll}
\hline Symbol & Description \\
\hline$\Lambda$ & Recruitment rate of susceptibles into the sexually active population \\
$\beta$ & Probability of transmission \\
$\mu$ & Natural mortality rate \\
$\delta$ & Disease-induced mortality rate \\
$\sigma$ & Rate at which aware infectives develop symptoms \\
$\theta$ & Rate at which unaware infectives develop symptoms \\
$\rho$ & Rate of status awareness due to screening method \\
$\alpha$ & Progression rate of pre-AIDS individuals to full blown AIDS \\
$v$ & Rate at which unaware infectives develop full blown AIDS \\
$\eta$ & Rate at which aware infectives develop full blown AIDS \\
$\tau$ & Emigration rate of pre-AIDS and AIDS patients \\
$\omega$ & Measure of the psychological or inhibitory effect \\
\hline
\end{tabular}

Lemma 3.1. The disease-free equilibrium $E_{0}$ of the basic model with standard incidence is locally asymptotically stable if $R_{0}^{\mathrm{st}}<1$ and unstable if $R_{0}^{\mathrm{st}}>1$.

Lemma 3.1 implies that the disease can be eliminated from the population if the initial size of the subpopulations are in the basin of attraction of the disease-free equilibrium $E_{0}$. The contributions of $I_{1}, I_{2}, P$, and $A$ are

$$
\begin{gathered}
R_{0}^{I_{1}}=\frac{\beta}{\rho+\theta+v+\mu+\tau}, \quad R_{0}^{I_{2}}=\frac{\beta k_{1}}{\sigma+\eta+\mu+\tau}, \\
R_{0}^{P}=\frac{\beta k_{2}}{\mu+\alpha}, \quad R_{0}^{A}=\frac{\beta k_{3}}{\delta+\mu^{\prime}}
\end{gathered}
$$

respectively, where $R_{0}^{I_{1}}$ is the contribution to the reproduction number by unaware infectives $I_{1}, R_{0}^{I_{2}}$ is the contribution to the reproduction number by aware infectives $I_{2}, R_{0}^{P}$ is the contribution to the reproduction number by pre-AIDS individuals $P$, and $R_{0}^{A}$ is the contribution to the reproduction number by AIDS individuals $A$. The terms in (3.3) can be interpreted as follows.

(i) $1 /(\rho+\theta+v+\mu+\tau), 1 /(\sigma+\eta+\mu+\tau), 1 /(\mu+\alpha)$, and $1 /(\delta+\mu)$ are the average times an individual spends in either of the following disease classes $I_{1}, I_{2}, P$, and $A$, respectively.

(ii) $\rho /(\rho+\theta+v+\mu+\tau)$ is the proportion of individuals who become aware of their infection by progression from compartment $I_{1}$ to $I_{2}$. 


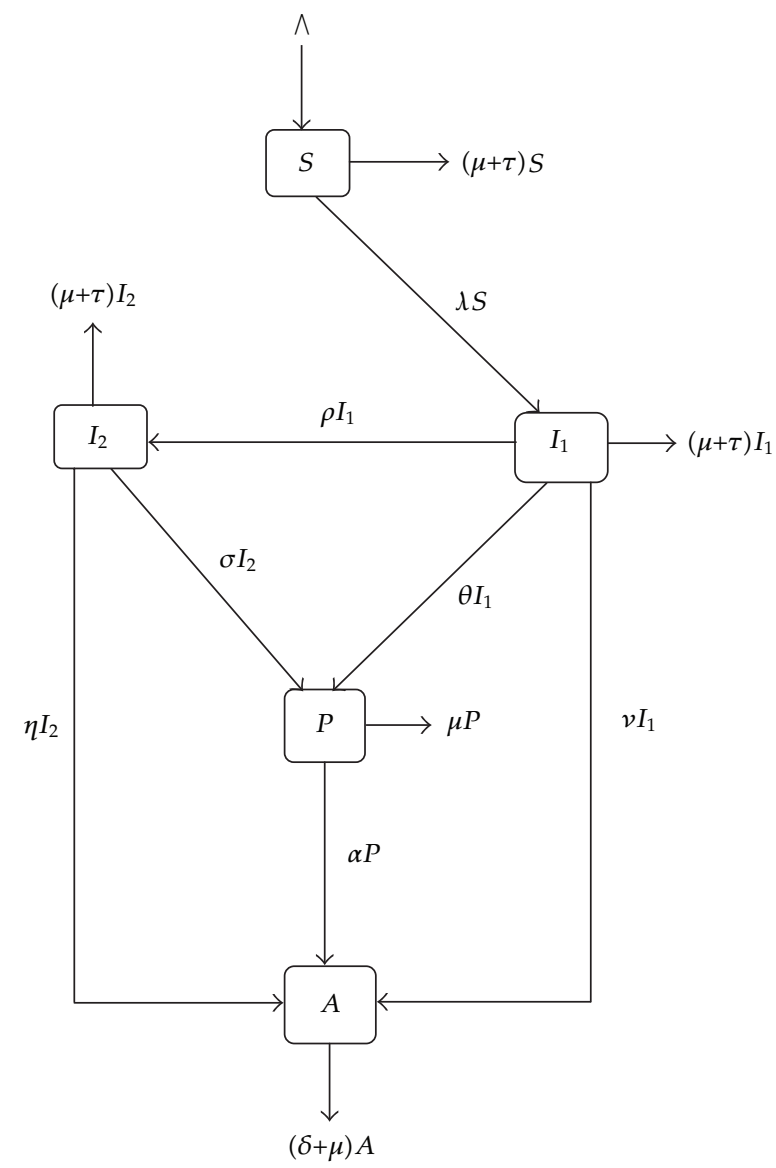

Figure 1: The basic model compartments and flow.

(iii) $\theta /(\rho+\theta+v+\mu+\tau)$ is the proportion of individuals who develop symptoms and progress from compartment $I_{1}$ to $P$.

(iv) $v /(\rho+\theta+v+\mu+\tau)$ is the proportion of individuals who develop full blown AIDS from compartment $I_{1}$.

(v) $\sigma /(\sigma+\eta+\mu+\tau)$ is the proportion of individuals who develop symptoms from compartment $I_{2}$.

(vi) $\eta /(\sigma+\eta+\mu+\tau)$ is the proportion of individuals who develop full blown AIDS from compartment $I_{2}$.

(vii) $\alpha /(\mu+\alpha)$ is the proportion of individuals who develop full blown AIDS from compartment $P$.

The endemic equilibrium is obtained by solving the following system:

$$
\begin{gathered}
\Lambda-\lambda_{\mathrm{st}}^{*} S^{*}-(\mu+\tau) S^{*}=0, \\
\lambda_{\mathrm{st}}^{*} S^{*}-(\rho+\theta+\nu+\mu+\tau) I_{1}^{*}=0, \\
\rho I_{1}^{*}-(\sigma+\eta+\mu+\tau) I_{2}^{*}=0 .
\end{gathered}
$$




$$
\begin{gathered}
\theta I_{1}^{*}+\sigma I_{2}^{*}-(\mu+\alpha) P^{*}=0, \\
v I_{1}^{*}+\eta I_{2}^{*}+\alpha P^{*}-(\delta+\mu) A^{*}=0,
\end{gathered}
$$

where

$$
\lambda_{\mathrm{st}}^{*}=\frac{\beta\left(I_{1}^{*}+k_{1} I_{2}^{*}+k_{2} P^{*}+k_{3} A^{*}\right)}{N^{*}} .
$$

From (3.1), the steady-state expressions for $I_{2}^{*}, P^{*}$, and $A^{*}$ in terms of $I_{1}^{*}$ are given by

$$
I_{2}^{*}=\omega_{1} I_{1}^{*}, \quad P^{*}=\omega_{2} I_{1}^{*}, \quad A^{*}=\omega_{3} I_{1}^{*},
$$

where

$$
\omega_{1}=\frac{\rho}{\sigma+\eta+\mu+\tau}, \quad \omega_{2}=\frac{\theta+\sigma \omega_{1}}{\mu+\alpha}, \quad \omega_{3}=\frac{v+\eta \omega_{1}+\alpha \omega_{2}}{\delta+\mu}
$$

From (3.7),

$$
\lambda_{\mathrm{st}}^{*}=\frac{\phi I_{1}^{*}}{N^{*}}, \quad \text { where } \phi=\beta+\beta k_{1} \omega_{1}+\beta k_{2} \omega_{2}+\beta k_{3} \omega_{3}
$$

Substituting for $\lambda_{\mathrm{st}}^{*}$ in the second equation of (3.1), we have either $I_{1}^{*}=0$ or

$$
\phi S^{*}-(\rho+\theta+\mathcal{v}+\mu+\tau) N^{*}=0 .
$$

The total population $N$ at steady state can be written as

$$
N^{*}=S^{*}+\omega_{4} I_{1}^{*}, \quad \text { with } \omega_{4}=1+\omega_{1}+\omega_{2}+\omega_{3} .
$$

Substituting for $N^{*}$ in (3.11), we obtain

$$
I_{1}^{*}=\left(\frac{\vartheta-1}{\omega_{4}}\right) S^{*}
$$

where $\vartheta=\phi /(\rho+\theta+v+\mu+\tau)$. After some little rearrangement, we obtain

$$
\vartheta=R_{0}^{\mathrm{st}} \text {. }
$$

Substituting (3.10) and (3.13) in the first equation of (3.1) gives

$$
S^{*}=\frac{\Lambda R_{0}^{\mathrm{st}} \omega_{4}}{\phi\left(R_{0}^{\mathrm{st}}-1\right)+R_{0}^{\mathrm{st}} \omega_{4}(\mu+\tau)} .
$$


The first case $I_{1}^{*}=0$ results in the disease-free equilibrium point. The second case gives the endemic equilibrium point $E^{*}=\left(S^{*}, I_{1}^{*}, I_{2}^{*}, P^{*}, A^{*}\right)$, where

$$
\begin{gathered}
S^{*}=\frac{\Lambda R_{0}^{\mathrm{st}} \omega_{4}}{\phi\left(R_{0}^{\mathrm{st}}-1\right)+R_{0}^{\mathrm{st}} \omega_{4}(\mu+\tau)}, \\
I_{1}^{*}=\left(\frac{R_{0}^{\mathrm{st}}-1}{\omega_{4}}\right)\left(\frac{\Lambda R_{0}^{\mathrm{st}} \omega_{4}}{\phi\left(R_{0}^{\mathrm{st}}-1\right)+R_{0}^{\mathrm{st}} \omega_{4}(\mu+\tau)}\right), \\
I_{2}^{*}=\omega_{1} I_{1}^{*}, \\
P^{*}=\omega_{2} I_{1}^{*}, \\
A^{*}=\omega_{3} I_{1}^{*} .
\end{gathered}
$$

We thus have the following result on the existence of the endemic equilibrium point.

Lemma 3.2. If $R_{0}^{\text {st }} \leq 1$, system (3.1) has a unique disease-free equilibrium $E_{0}$. If $R_{0}^{\text {st }}>1$, there exists a unique endemic equilibrium point $E^{*}$ whose coordinates are given by (3.16).

\subsubsection{Local Stability of the Endemic Equilibrium}

Here, the center manifold approach [14] as described by Theorem 4.1 in Castillo-Chavez and Song [15] will be used. To apply the said theorem, it is intuitive to rewrite system (3.1) after a change of variables: $S=x_{1}, I_{1}=x_{2}, I_{2}=x_{3}, P=x_{4}, A=x_{5}$. In vector form, system (3.1) takes the form $d X / d t=f(X)$, where $X=\left[x_{1}, x_{2}, x_{3}, x_{4}, x_{5}\right]^{T}$ and $[\cdot]^{T}$ denotes the matrix transpose. We can thus write the system as

$$
\begin{gathered}
\frac{d x_{1}}{d t}=f_{1}=\Lambda-\beta\left(\frac{x_{2}+k_{1} x_{3}+k_{2} x_{4}+k_{3} x_{5}}{x_{1}+x_{2}+x_{3}+x_{4}+x_{5}}\right) x_{1}-(\mu+\tau) x_{1} \\
\frac{d x_{2}}{d t}=f_{2}=\beta\left(\frac{x_{2}+k_{1} x_{3}+k_{2} x_{4}+k_{3} x_{5}}{x_{1}+x_{2}+x_{3}+x_{4}+x_{5}}\right) x_{1}-(\rho+\theta+v+\mu+\tau) x_{2} \\
\frac{d x_{3}}{d t}=f_{3}=\rho x_{2}-(\sigma+\eta+\mu+\tau) x_{3} \\
\frac{d x_{4}}{d t}=f_{4}=\theta x_{2}+\sigma x_{3}-(\mu+\alpha) x_{4} \\
\frac{d x_{5}}{d t}=f_{5}=v x_{2}+\eta x_{3}+\alpha x_{4}-(\delta+\mu) x_{5} .
\end{gathered}
$$

The Jacobian matrix of system (3.17) at the disease-free equilibrium is given by

$$
\mathrm{J}_{\mathrm{E} 0}=\left[\begin{array}{ccccc}
-(\mu+\tau) & -\beta & -\beta k_{1} & -\beta k_{2} & -\beta k_{3} \\
0 & \beta-E_{1} & \beta k_{1} & \beta k_{2} & \beta k_{3} \\
0 & \rho & -B & 0 & 0 \\
0 & \theta & \sigma & -C & 0 \\
0 & v & \eta & \alpha & -D
\end{array}\right] .
$$


Suppose that $\beta=\beta^{*}$ is chosen as a bifurcation parameter. Consider the case when $R_{0}^{\text {st }}=1$, then solving for $\beta$ from $R_{0}^{\text {st }}=1$ gives

$$
\beta=\beta^{*}=\frac{E_{1} B C D}{B C D+k_{1} \rho C D+k_{2} D(\sigma \rho+\theta B)+k 3(\alpha(\sigma \rho+\theta B)+C(\eta \rho+v B))} .
$$

We calculate the right and left eigenvectors associated with $J_{E 0}$. Note that 0 is a simple eigenvalue of $J_{E 0}$. From (3.18), we obtain the following equations:

$$
\begin{gathered}
-(\mu+\tau) v_{1}-\beta v_{2}-\beta k_{1} v_{3}-\beta k_{2} v_{4}-\beta k_{3} v_{5}=0 \\
\left(\beta-E_{1}\right) v_{2}+\beta k_{1} v_{3} \beta k_{2} v_{4}+\beta k_{3} v-5=0 \\
\rho v_{2}-B v_{3}=0 \\
\theta v_{2}+\sigma v_{3}-C v_{4}=0 \\
v v_{2}+\eta v_{3}+\alpha v_{4}-D v_{5}=0
\end{gathered}
$$

The Jacobian matrix (3.18) has a right eigenvector $V=\left[v_{1}, v_{2}, v_{3}, v_{4}, v_{5}\right]^{T}$ associated with the zero eigenvalue given by

$$
v_{1}=-z_{1} v_{3}, \quad v_{2}=\frac{B v_{3}}{\rho}, \quad v_{3}=v_{3}>0, \quad v_{4}=z_{1} v_{3}, \quad v_{5}=z_{2} v_{3},
$$

where

$$
z_{1}=\frac{\theta B+\sigma \rho}{C \rho}, \quad z_{2}=\frac{v B+\eta \rho+\alpha \rho z_{1}}{D \rho}
$$

$B, C$, and $D$ are defined in (3.4). We then transpose the Jacobian matrix (3.18) to calculate the left eigenvector

$$
\left(\mathrm{J}_{\mathrm{E} 0}\right)^{\mathrm{T}}=\left[\begin{array}{ccccc}
-(\mu+\tau) & 0 & 0 & 0 & 0 \\
-\beta & \beta-E_{1} & \rho & \theta & \nu \\
-\beta k_{1} & \beta k_{1} & -B & \sigma & \eta \\
-\beta k_{2} & \beta k_{2} & 0 & -C & \alpha \\
-\beta k_{3} & \beta k_{3} & 0 & 0 & -D
\end{array}\right],
$$


which leads to the following system of equations:

$$
\begin{gathered}
-(\mu+\tau) \eta_{1}=0 \\
-\beta \eta_{1}+\left(\beta-E_{1}\right) \eta_{2}+\rho \eta_{3}+\theta \eta_{4}+v \eta_{5}=0 \\
-\beta k_{1} \eta_{1}+\beta k_{1} \eta_{2}-B \eta_{3}+\sigma \eta_{4}+\eta \eta_{5}=0 \\
-\beta k_{2} \eta_{1}+\beta k_{2} \eta_{2}-C \eta_{4}+\alpha \eta_{5}=0 \\
-\beta k_{3}+\beta k_{3}-D=0 .
\end{gathered}
$$

Thus, solving system (3.24) yields the following left eigenvector $\eta=\left[\eta_{1}, \eta_{2}, \eta_{3}, \eta_{4}, \eta_{5}\right]^{T}$ associated with the zero eigenvalue, where

$$
\begin{gathered}
\eta_{1}=0, \quad \eta_{2}=\frac{-z_{5} D \eta_{3}}{z_{4} \beta k_{3}}, \quad \eta_{3}=\eta_{3}>0, \quad \eta_{4}=z_{6} \eta_{3}, \quad \eta_{5}=\frac{-z_{5} \eta_{3}}{z_{4}}, \\
z_{4}=\frac{\sigma\left(\left(\beta-E_{1}\right) D+v \beta k_{3}\right)-\theta\left(\beta k_{1} D+\eta \beta k_{3}\right)}{\sigma \beta k_{3}}, \quad z_{5}=\frac{\sigma \rho+\theta B}{\sigma}, \\
z_{6}=\frac{B \beta k_{3}+\left(\beta k_{1} D+\eta \beta k_{3}\right) z_{5}}{z_{4} \sigma \beta k_{3}} .
\end{gathered}
$$

The local bifurcation analysis near $\beta=\beta^{*}$ is then determined by the signs of two associated constants, denoted herein by $\psi_{1}$ and $\psi_{2}$. In general, using $\beta$ as the bifurcation parameter ensures that $\psi_{2}>0$ [13], and, for this reason, the expression for $\psi_{2}$ is not derived. $\psi_{1}$ is given by

$$
\psi_{1}=\frac{z_{5} D \eta_{3}(\mu+\tau) \sigma \beta k_{3} v_{3}^{2}}{\Lambda k_{3}\left(\sigma \beta D+\sigma v \beta k_{3}-\left(\sigma E_{1} D+\theta\left(\beta k_{1} D+\eta \beta k_{3}\right)\right)\right)}\left(g_{1}+g_{2}+g_{3}+g_{4}\right),
$$

where

$$
\begin{gathered}
g_{1}=\frac{1}{\rho}\left[\frac{2 \beta}{\rho}+1+k_{1}+\left(1+k_{2}\right) z_{1}+\left(1+k_{3}\right) z_{2}\right], \\
g_{2}=\frac{\left(1+k_{1}\right) \beta}{\rho}+2 k_{1}+\left(k_{1}+k_{2}\right) z_{1}+\left(k_{1}+k_{3}\right) z_{2}, \\
g_{3}=z_{1}\left[\left(1+\beta_{2}\right) \frac{\beta}{\rho}+k_{1}+k_{2}+k_{2} z_{1}+\left(k_{2}+k_{3}\right) z_{2}\right], \\
g_{4}=z_{2}\left[\frac{\left(1+k_{3}\right) \beta}{\rho}+k_{1}+k_{3}+\left(k_{2}+k_{3}\right) z_{1}+2 k_{2} z_{2}\right],
\end{gathered}
$$

and $z_{1}, z_{2}, z_{5}$ are defined in (3.22) and (3.25). It can be shown that $\psi_{1}<0$ if the following inequality holds:

$$
\sigma \beta D+\sigma v \beta k_{3}<\sigma E_{1} D+\theta\left(\beta k_{1} D+\eta \beta k_{3}\right) .
$$


Since $\psi_{1}<0$, this precludes the phenomenon of backward bifurcation, and, consequently, local and global stability follows, in which case elimination of the disease may be guaranteed via the available control measures. From the above mentioned, the following results are established.

Theorem 3.3. The endemic equilibrium $E^{*}$ is locally asymptotically stable if $R_{0}^{\text {st }}>1$ and unstable otherwise.

Theorem 3.4. The disease-free equilibrium of the basic model with standard incidence rate is globally asymptotically stable if $R_{0}^{\text {st }} \leq 1$.

\subsection{The Basic Model with Saturated Incidence}

The basic model with saturated incidence has the same disease-free equilibrium as the standard incidence model given by (3.2). Using the next generation operator method as described by van den Driessche and Watmougth [13] and the notation therein, the average number of new infections generated by a single infected individual in a completely susceptible population [16] for model system (3.1) is

$$
R_{0}^{\mathrm{sat}}=\gamma_{6} R_{0}^{I_{1}}+\gamma_{7} R_{0}^{I_{2}}+\gamma_{8} R_{0}^{P}+\gamma_{9} R_{0}^{A}
$$

where

$$
\begin{gathered}
R_{0}^{I_{1}}=\frac{\beta}{\rho+\theta+v+\mu+\tau}, \quad R_{0}^{I_{2}}=\frac{\beta k_{1}}{\sigma+\eta+\mu+\tau}, \quad R_{0}^{P}=\frac{\beta k_{2}}{\mu+\alpha}, \\
R_{0}^{A}=\frac{\beta k_{3}}{\delta+\mu}, \quad \gamma_{6}=\frac{\Lambda}{\mu+\tau}, \quad \gamma_{7}=\frac{\rho \gamma_{6}}{E_{1}}, \\
\gamma_{8}=\frac{(\sigma \rho+\theta B) \gamma_{6}}{E_{1} B}, \quad \gamma_{9}=\frac{(\alpha(\sigma \rho+\theta B)+C(\eta \rho+v B)) \gamma_{6}}{E_{1} B C},
\end{gathered}
$$

with the expressions of $E_{1}, B, C, D$ given by (3.4). Equation (3.29) can be rewritten as

$$
R_{0}^{\mathrm{sat}}=\frac{\Lambda}{\mu+\tau} R_{0}^{\mathrm{st}}
$$

The reproduction number for the saturated incidence model is greater than that for the standard incidence model, that is, $R_{0}^{\text {sat }}>R_{0}^{\text {st }}$ since $\Lambda>(\mu+\tau)$; hence, the saturated incidence may overestimate the number of secondary infection compared to the same outcome when the standard incidence formulation is used. From Theorem 2 in van den Driessche and Watmough [13] and the fact that the disease-free equilibrium is the same as in (3.2), the following result holds.

Lemma 3.5. The disease-free equilibrium (3.2) of the basic model with saturated incidence rate is both locally and globally asymptotically stable if $R_{0}^{\text {sat }}<1$ and unstable otherwise. 
It can be shown that the saturated incidence model has no endemic equilibrium when $R_{0}^{\text {sat }} \leq 1$. Thus, we claim the following result.

Theorem 3.6. The disease-free equilibrium of the basic model with saturated incidence is globally asymptotically stable if $R_{0}^{\text {sat }} \leq 1$.

The consequence of Theorem 3.6 vis-a-vis backward bifurcation is that the saturated incidence model does not exhibit backward bifurcation; consequently, the endemic equilibrium is unique and is globally asymptotically stable whenever $R_{0}^{\text {sat }}>1$. This concludes the analysis of the basic models. The next section extends these models by incorporating some control measures.

\section{HIV/AIDS Model with Control Measures}

Three control measures, namely, public health educational campaigns, condom use, and treatment of infected individuals, are incorporated into the basic model (3.1). In the following, we describe the additional variables and parameters added to the extended model. Educated and individuals under treatment are denoted by the variables $E(t)$ and $T(t)$, respectively. Susceptible individuals are recruited into the sexually active population at the rate, $\Lambda$, a proportion $b$ of which is assumed to be educated but susceptibles and move to the educated class, $E$. The complementary proportion, $(1-b)$, is susceptible and moves to the susceptible class, $S$, who is educated at a constant rate, $r$, and moves into the $E$ class. Susceptible individuals acquire infection upon effective contact with an infected individual at the rate $\lambda^{0}$ and move to the class $I_{1}$ of infected individuals who are not aware of their infection. Educated individuals are infected at the rate $(1-k) \lambda^{0}$ and move to the class $I_{1}$, where $k$ measures the overall effectiveness of the public health educational campaign, $0<k<1$. The two extreme values are excluded because $k=0$ implies that education is useless, while $k=1$ implies that education is completely effective.

A proportion $p$ of individuals use condoms. Since individuals in the $I_{2}$-class are aware of their infection, they seek treatment at the rate $\rho_{1}$. Infected individuals who have developed symptoms of the disease seek treatment at the rate, $\rho_{2}$. Individuals in the $T$ class interact with the rest of the community, but they are less infectious than those in the $I_{1}$ class, because the use of treatment significantly reduces the viral load [5]. $k_{4}$ is the relative infectivity of individuals in the $I_{1}$ class and $0<k_{4}<1$ ( 0 means the drug is useless, and 1 means the drug is completely effective in stopping HIV transmission). They develop full blown AIDS at the rate $\alpha_{1}$ with $0<\alpha_{1}<1$ and move to the AIDS class $A$. Further, there is a constant emigration of educated susceptibles and those on treatment at the rate $\tau$. From the aforesaid, the forces of infection are now defined as

$$
\lambda_{\mathrm{st}}^{0}=\frac{\beta\left(I_{1}+k_{1} I_{2}+k_{2} P+k_{4} T+k_{3} A\right)}{N}, \quad \lambda_{\mathrm{sat}}^{0}=\frac{\beta\left(I_{1}+k_{1} I_{2}+k_{2} P+k_{4} T+k_{3} A\right)}{1+\omega\left(I_{1}+I_{2}+P+T+A\right)}
$$

for the standard incidence and saturated incidence models, respectively, with $k_{4}<k_{1}<k_{2}<$ $k_{3}$. The model flow chart is shown in Figure 2. The additional state variables and parameters are described, respectively, in Tables 3 and 4 . The above and previous assumptions lead 


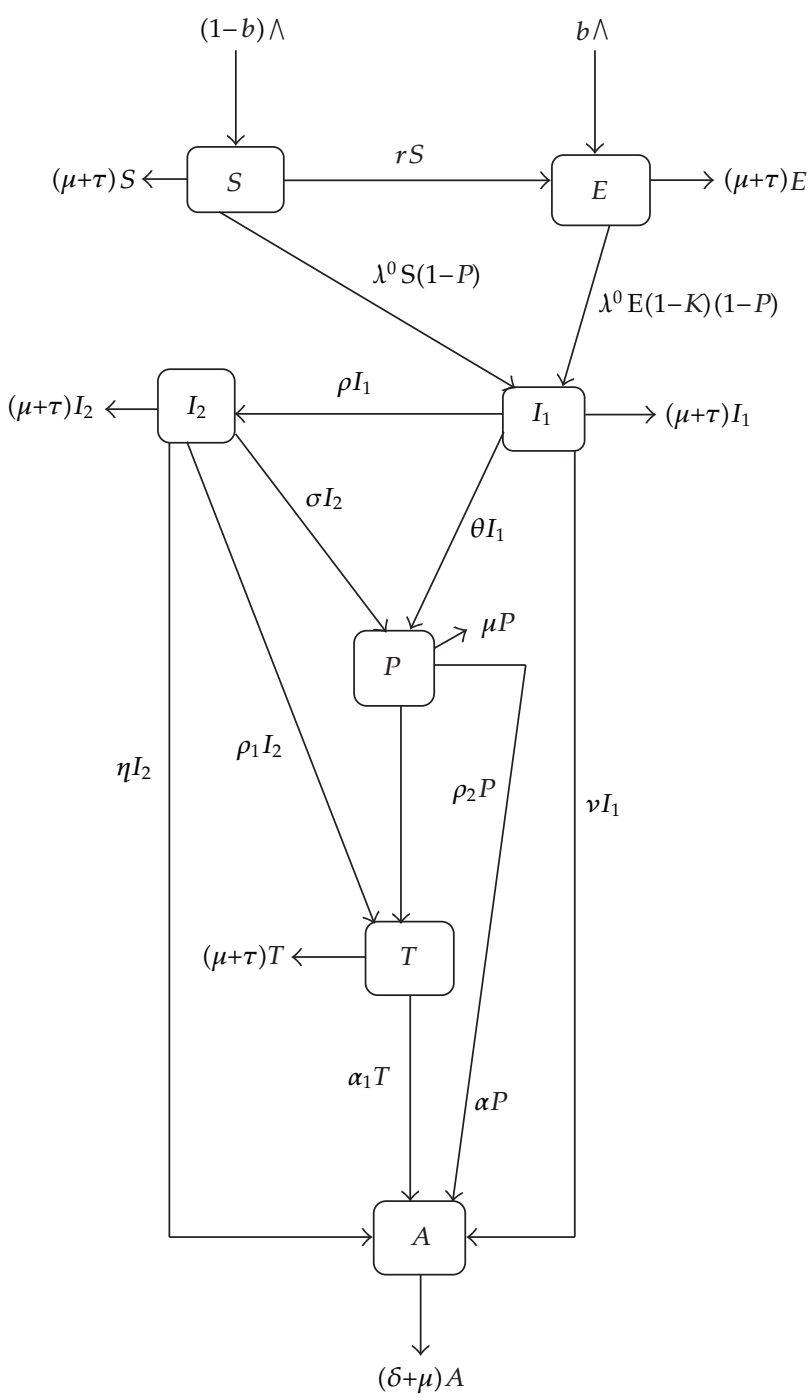

Figure 2: HIV / AIDS model structure with control measures.

to the following system of nonlinear ordinary differential equations describing the disease dynamics when control measures are implemented:

$$
\begin{gathered}
\frac{d S}{d t}=(1-b) \Lambda-\lambda^{0} S(1-p)-(r+\mu+\tau) S, \\
\frac{d E}{d t}=b \Lambda+r S-\lambda^{0} E(1-k)(1-p)-(\mu+\tau) E, \\
\frac{d I_{1}}{d t}=\lambda^{0} S(1-p)+\lambda^{0} E(1-k)(1-p)-(\rho+v+\theta+\mu+\tau) I_{1},
\end{gathered}
$$




$$
\begin{gathered}
\frac{d I_{2}}{d t}=\rho I_{1}-\left(\sigma+\rho_{1}+\eta+\mu+\tau\right) I_{2}, \\
\frac{d P}{d t}=\theta I_{1}+\sigma I_{2}-\left(\rho_{2}+\mu+\alpha\right) P, \\
\frac{d T}{d t}=\rho_{1} I_{2}+\rho_{2} P-\left(\alpha_{1}+\mu+\tau\right) T, \\
\frac{d A}{d t}=v I_{1}+\eta I_{2}+\alpha P+\alpha_{1} T-(\delta+\mu) A,
\end{gathered}
$$

with initial conditions $S(0)=S^{0}, E(0)=E^{0}, I_{1}(0)=I_{1}^{0}, I_{2}(0)=I_{2}^{0}, P(0)=P^{0}, T(0)=$ $T^{0}, A(0)=A^{0}$.

\subsection{Model with Standard Incidence}

The disease-free equilibrium $E_{02}$ of (4.2) is

$$
E_{02}=\left\{\frac{(1-b) \Lambda}{r+\mu+\tau}, \frac{b \Lambda(\mu+\tau)+\Lambda r}{(\mu+\tau)(r+\mu+\tau)}, 0,0,0,0,0\right\} .
$$

Again, using the next generation matrix operator [13], the control measure (public health educational campaigns, condom use, and treatment) induced effective reproduction number of the model with standard incidence denoted by $R_{e}^{\text {st }}$ is given by

$$
R_{e}^{\mathrm{st}}=\gamma_{1} R_{e}^{I_{1}}+\gamma_{2} R_{e}^{I_{2}}+\gamma_{3} R_{e}^{P}+\gamma_{4} R_{e}^{T}+\gamma_{5} R_{e}^{A}
$$

where

$$
\begin{gathered}
R_{e}^{I_{1}}=\frac{\beta}{\rho+\theta+v+\mu+\tau}, \quad R_{e}^{I_{2}}=\frac{\beta k_{1}}{\sigma+\rho_{1}+\eta+\mu+\tau}, \quad R_{e}^{P}=\frac{\beta k_{2}}{\rho_{2}+\mu+\alpha}, \\
R_{e}^{T}=\frac{\beta k_{4}}{\alpha_{1}+\mu+\tau}, \quad R_{e}^{A}=\frac{\beta k_{3}}{\delta+\mu}, \\
\gamma_{1}=(1-p)\left(\frac{(1-b)(\mu+\tau)+(b(\mu+\tau)+r)(1-k)}{r+\mu+\tau}\right), \quad \gamma_{2}=\frac{\gamma_{1} \rho}{E_{1}}, \\
\gamma_{5}=\frac{\gamma_{1}\left(\rho_{1} \rho C_{1}+\rho_{2} \theta B_{1}+\rho_{2} \sigma \rho\right)}{E_{1} B_{1} C_{1}}, \quad \gamma_{3}=\frac{\gamma_{1}\left(\theta B_{1}+\sigma \rho\right)}{E_{1} B_{1}}, \\
C_{1}=C+\rho_{2}, \quad l=\alpha_{1}+\mu+\tau .
\end{gathered}
$$


Table 3: Additional state variables of the model with control measures.

\begin{tabular}{ll}
\hline Symbol & Description \\
\hline$E(t)$ & Educated individuals at time $t$ \\
$T(t)$ & Treated individuals at time $t$ \\
\hline
\end{tabular}

Table 4: Additional parameters of the model with control measures.

\begin{tabular}{ll}
\hline Symbol & Description \\
\hline$b$ & Proportion of educated individuals \\
$k$ & Education effectiveness \\
$p$ & Proportion of individuals who use condoms \\
$r$ & Rate at which susceptible individuals are educated \\
$\rho_{1}$ & Rate at which aware infectives seek treatment \\
$\rho_{2}$ & Rate at which pre-AIDS/asymptomatic individuals seek treatment \\
$\alpha_{1}$ & Rate at which treated individuals develop full blown AIDS \\
$\tau$ & Emigration rate of educated and treated patients \\
\hline
\end{tabular}

$B, C$, and $D$ are defined in (3.4) above. $R_{e}^{I_{1}}$ is the contribution to the effective reproduction number by unaware infectives $I_{1}, R_{e}^{I_{2}}$ is the contribution to the effective reproduction number by aware infectives $I_{2}, R_{e}^{P}$ is the contribution to the effective reproduction number by pre-AIDS individuals $P, R_{e}^{T}$ is the contribution to the effective reproduction number by individuals who are under treatment $T$, and $R_{e}^{A}$ is the contribution to the effective reproduction number by individuals with full blown AIDS. When there are no control measures, that is $b=r=k=p=\rho_{1}=\rho_{2}=\alpha_{1}=0, R_{e}^{\text {st }}$ reduces to $R_{0}^{\text {st }}$ whose expression appears in (3.3). Thus, from Theorem 2 in van de Driessche and Watmough [13], we claim the following result.

Lemma 4.1. The disease-free equilibrium $E_{02}$ of the model with control measures and standard incidence is locally asymptotically stable whenever $R_{e}^{\text {st }}<1$ and unstable otherwise.

The threshold quantity $R_{e}^{\text {st }}$ is the reproduction number for the standard incidence model which measures the average number of new HIV infections generated by a single HIV-infected individual in a population where some individuals are educated, a proportion using condoms, and others are receiving treatment.

\section{Existence of the endemic equilibrium}

At steady state, the variables of the standard incidence model can be expressed in terms of $\lambda_{\mathrm{st}}^{0 * *}$ as follows:

$$
\begin{gathered}
S^{* *}=\frac{(1-b) \Lambda}{\lambda_{\mathrm{st}}^{0 * *}(1-p)+(r+\mu+\tau)}, \quad I_{1}^{* *}=\frac{j \lambda_{\mathrm{st}}^{0 * *}(1-p)}{E_{1}}, \\
E^{* *}=\frac{b \Lambda}{\lambda_{\mathrm{st}}^{0 * *} d+e}+\frac{r a}{\left(\lambda_{\mathrm{st}}^{0 * *} d+e\right)\left(\lambda_{\mathrm{st}}^{0 * *}(1-p)+c\right)}, \quad I_{2}^{* *}=\frac{j \rho \lambda_{\mathrm{st}}^{0 * *}(1-p)}{B_{1} E_{1}},
\end{gathered}
$$


ISRN Applied Mathematics

$$
\begin{gathered}
P^{* *}=\frac{j f_{2} \lambda_{\mathrm{st}}^{0 * *}(1-p)}{E_{1}}, \quad T^{* *}=\frac{j f_{3} \lambda_{\mathrm{st}}^{0 * *}(1-p)}{E_{1}}, \\
A^{* *}=\frac{j f_{4} \lambda_{\mathrm{st}}^{0 * *}(1-p)}{D E_{1}},
\end{gathered}
$$

where

$$
\begin{gathered}
\lambda_{\mathrm{st}}^{0 * *}=\frac{\beta\left(I_{1}^{*}+k_{1} I_{2}^{*}+k_{2} P^{*}+k_{4} T^{*}+k_{3} A^{*}\right)}{N^{*}}, \\
j=\left\{\frac{a}{\lambda_{\mathrm{st}}^{* *}(1-p)+c}+\frac{f_{1}}{\lambda_{\mathrm{st}}^{0 * *} d+e}+\frac{d r \Lambda}{\left(\lambda_{\mathrm{st}}^{* * *}(1-p)+c\right)\left(\lambda_{\mathrm{st}}^{0 * *} d+e\right)}\right\}, \\
a=(1-b) \Lambda, \quad c=r+\mu+\tau, \\
d=(1-k)(1-p), \quad e=\mu+\tau, \\
f_{1}=(1-k) b \Lambda, \quad f_{2}=\frac{\theta}{C_{1}}+\frac{\sigma \rho}{C_{1} B_{1}}, \\
f_{3}=\frac{\rho \rho_{1}}{l B_{1}}+\frac{\rho_{2}}{l} f_{2}, \quad f_{4}=v+\frac{\eta \rho}{B_{1}}+\alpha f_{2}+\alpha_{1} f_{3}, \\
l=\alpha_{1}+\mu+\tau .
\end{gathered}
$$

Substituting (4.6) into the expression for $\lambda_{\mathrm{st}}^{0 * *}$ at steady state and after a little rearrangement, we obtain

$$
g\left(\lambda_{\mathrm{st}}^{0 * *}\right)=b_{11} \lambda_{\mathrm{st}}^{0 * * 2}+b_{21} \lambda^{0 * *}+b_{31}=0
$$

where

$$
\begin{gathered}
b_{11}=f_{5} a b_{1} d+f_{1} f_{5} b_{1}^{2}, \\
b_{21}=a E_{1} d+b_{1} b \Lambda E_{1}+f_{5} b_{1} a e+f_{5} f_{1} b_{1} c+f_{5} b_{1} d r \Lambda-\left(a b_{1} d f_{6}+f_{6} f_{1} b_{1}^{2}\right), \\
b_{31}=-b_{1} d r \Lambda f_{6}+r a E_{1}+a E_{1} c+b \Lambda E_{1} c\left(1-\frac{R_{e}^{\mathrm{st}}}{b}\right), \\
f_{5}=1+\frac{\rho}{B_{1}}+f_{2}+f_{3}+\frac{f_{4}}{D}, \quad f_{6}=\beta+\frac{\beta k_{1} \rho}{B_{1}}+\beta k_{2} f_{2}+\beta k_{4} f_{3}+\frac{\beta k_{3} f_{4}}{D} .
\end{gathered}
$$

Both $b_{11}>0$ and $b_{21}>0$ if $a b_{1} d f_{6}+f_{6} f_{1} b_{1}^{2}<a E_{1} d+b_{1} b \Lambda E_{1}+f_{5} b_{1} a e+f_{5} f_{1} b_{1} c+f_{5} b_{1} d r \Lambda$, while $b_{31}>0$ if $R_{e}^{\text {st }}<1$ and $r a E_{1}+a E_{1} c+b \Lambda E_{1} c\left(1-\left(R_{e}^{\text {st }} / b\right)\right)>b_{1} d r \Lambda f_{6}$. By the RouthHurwiz criterion, the quadratic (4.8) has no positive root; hence, no endemic equilibrium exists when $R_{e}^{\text {st }}<1$. When $R_{e}^{\text {st }}=1, b_{31}>0$ if $b_{1} d r \Lambda f_{6}+\Lambda E_{1} c<r a E_{1}+a E_{1} e+b \Lambda E_{1} c$, then by the Routh-Hurwitz criterion, the quadratic (4.8) has no positive root; thus, no endemic 
equilibrium exists when $R_{e}^{\text {st }}=1$. The case when $R_{e}^{\text {st }}>1$ makes $b_{31}<0$ if $b_{1} d r \Lambda f_{6}+b \Lambda E_{1} c(1-$ $\left.\left(R_{e}^{\mathrm{st}} / b\right)\right)>r a E_{1}+a E_{1} e$; in this case, the quadratic (4.8) has two roots with opposite signs (the negative root is epidemiologically meaningless). Hence, the following results are established.

Lemma 4.2. The standard incidence model has no endemic equilibrium when $R_{e}^{\text {st }} \leq 1$.

Theorem 4.3. If $R_{e}^{\text {st }} \leq 1$, the disease-free equilibrium is globally asymptotically stable and unstable if $R_{e}^{\mathrm{st}}>1$.

\subsection{Model with Saturated Incidence}

This model has the same disease-free equilibrium $E_{02}$ (see (4.3)) as that of the model with standard incidence. Using the next generation operator method [13], the effective reproduction number of the model with saturated incidence when the control measures are in place is given by

$$
R_{e}^{\mathrm{sat}}=\frac{\Lambda R_{e}^{\mathrm{st}}}{\mu+\tau} .
$$

Similar to the qualitative result obtained in the basic model analysis, the effective reproduction number for the saturated incidence is greater than that of standard incidence. That is $R_{e}^{\text {sat }}>R_{e}^{\text {st }}$ since $\Lambda>(\mu+\tau)$. In the absence of public health educational campaigns, condom use, and treatment (that is, $b=r=k=p=\rho_{1}=\rho_{2}=\alpha_{1}=0$ ), $R_{e}^{\text {sat }}$ simply reduces to $R_{0}^{\text {sat }}$. Hence, from Theorem 2 in [13], the following result is established.

Lemma 4.4. The disease-free equilibrium $E_{02}$ of the HIV/AIDS model with control measures and saturated incidence is locally asymptotically stable for $R_{e}^{\text {sat }}<1$ and unstable otherwise.

The threshold quantity $R_{e}^{\text {sat }}$ is the reproduction number for the saturated incidence model which measures the average number of new HIV infections generated by a single HIVinfected individual in a population where the three control measures (condom use, education and treatment) are implemented concurrently.

\section{Existence of endemic equilibria}

At the endemic steady state, the variables of the saturated incidence model can be expressed in terms of $\lambda_{\text {sat }}^{0 * *}$ as follows:

$$
\begin{gathered}
S^{* *}=\frac{(1-b) \Lambda}{\lambda_{\mathrm{sat}}^{0 * *}(1-p)+(r+\mu+\tau)}, \quad I_{1}^{* *}=\frac{j_{2} \lambda_{\mathrm{sat}}^{0 * *}(1-p)}{E_{1}}, \\
E^{* *}=\frac{b \Lambda}{\lambda_{\mathrm{sat}}^{0 * *} d+e}+\frac{r a}{\left(\lambda_{\mathrm{sat}}^{0 * *} d+e\right)\left(\lambda_{\mathrm{sat}}^{0 * *}(1-p)+c\right)}, \quad I_{2}^{* *}=\frac{j_{2} \rho \lambda_{\mathrm{sat}}^{0 * *}(1-p)}{B_{1} E_{1}},
\end{gathered}
$$


ISRN Applied Mathematics

$$
\begin{gathered}
P^{* *}=\frac{j_{2} f_{2} \lambda_{\mathrm{sat}}^{0 * *}(1-p)}{E_{1}}, \quad T^{* *}=\frac{j_{2} f_{3} \lambda_{\mathrm{sat}}^{0 * *}(1-p)}{E_{1}}, \\
A^{* *}=\frac{j_{2} f_{4} \lambda_{\mathrm{sat}}^{0 * *}(1-p)}{F E_{1}},
\end{gathered}
$$

where

$$
\begin{gathered}
j_{2}=\left\{\frac{a}{\lambda_{\mathrm{sat}}^{0 * *}(1-p)+c}+\frac{f_{1}}{\lambda_{\mathrm{sat}}^{0 * *} d+e}+\frac{d r \Lambda}{\left(\lambda_{\mathrm{sat}}^{0 * *}(1-p)+c\right)\left(\lambda_{\mathrm{sat}}^{0 * *} d+e\right)}\right\}, \\
\lambda_{\mathrm{sat}}^{0 * *}=\frac{\beta\left(I_{1}^{*}+k_{1} I_{2}^{*}+k_{2} P^{*}+k_{4} T^{*}+k_{3} A^{*}\right)}{1+\omega\left(I_{1}^{*}+I_{2}^{*}+P^{*}+T^{*}+A^{*}\right)},
\end{gathered}
$$

and $a, c, d, e, f_{1}, f_{2}, f_{3}, f_{4}, f_{5}, f_{6}, B_{1}, C_{1}, l, D$ are as defined in (4.7). Substituting (4.2) into the expression for $\lambda_{\text {sat }}^{0}$ (at steady state) and after some rearrangements, we obtain

$$
z_{1}\left(\lambda_{\mathrm{sat}}^{0 * *}\right)=a_{1} \lambda_{\mathrm{sat}}^{0 * * 2}+a_{2} \lambda_{\mathrm{sat}}^{0 * *}+a_{3}=0,
$$

where

$$
\begin{aligned}
a_{1}= & E_{1} d(1-p)+\omega f_{5} a d(1-p)+\omega f_{5} f_{1}(1-p)^{2}, \\
a_{2}= & E_{1} c(1-p)+E_{1} e(1-p)+\omega f_{5} a(1-p)+\omega f_{5} f_{1} c(1-p) \\
& +\omega f_{5} d r \Lambda(1-p)-\left(f_{6} a d(1-p)+f_{6} f_{1}(1-p)^{2}\right), \\
a_{3}= & E_{1} e c\left(1-R_{e}^{\mathrm{sat}}\right)-f_{6} d r \Lambda(1-p) .
\end{aligned}
$$

It is evident that $a_{1}>0, a_{2}>0$ if $f_{6} a d(1-p)+f_{6} f_{1}(1-p)^{2}<E_{1} c(1-p)+E_{1} e(1-p)+\omega f_{5} a(1-p)+$ $\omega f_{5} f_{1} c(1-p)+\omega f_{5} d r \Lambda(1-p)$, while $a_{3}>0$ if $R_{e}^{\text {sat }}<1$ and $f_{6} d r \Lambda(1-p)<E_{1} e c\left(1-R_{e}^{\text {sat }}\right)$. Thus, by the Routh-Hurwitz criterion, the quadratic in (4.13) has no positive root. The case $R_{e}^{\text {sat }}=1$ implies $a_{3}<0$, and, therefore, the quadratic in (4.13) now reads $a_{1} \lambda_{\text {sat }}^{0 * * 2}+a_{2} \lambda_{\text {sat }}^{0 * *}-a_{3}=0$, so that $\lambda_{\text {sat }}^{0 * *}=\left(-b \pm \sqrt{b^{2}+4 a c}\right) / 2 a$, where $a=a_{1}, b=a_{2}$, and $c=-a_{3}$. For $R_{e}^{\text {sat }}>1, a_{3}<0$, in this case, the quadratic has two roots with opposite signs (the negative root is biologically meaningless, hence the uniqueness of the endemic equilibrium). Thus, we have established the following results.

Lemma 4.5. The saturated incidence model has no endemic equilibrium when $R_{e}^{\mathrm{sat}}<1$.

Theorem 4.6. If $R_{e}^{\text {sat }}<1$, the disease-free equilibrium is globally asymptotically stable and unstable if $R_{e}^{\mathrm{sat}}>1$.

As a consequence of Theorem 4.6, the use of appropriate control measures can greatly minimize the outbreak burden and eliminate the disease in the community. The figures 
are generated using MatLab. In an uncontrolled outbreak, the basic reproduction number is significantly greater than the effective disease threshold number. In both basic and full models, we started the simulation at $t_{0}=0$ signifying the start of implementation of control strategies. The model demographic/epidemiological data are tabulated in Table 5. The initial conditions used in the model simulations are $S^{0}=100,000 ; E^{0}=70,000 ; I_{1}^{0}=50,000 ; I_{2}^{0}=$ 30,$000 ; P^{0}=35,000 ; T^{0}=20,000 ; A^{0}=25,000$. Some of the parameter values are assumed for the purpose of illustration.

\section{Dynamics of the basic model}

We simulate the basic model (3.1) with standard incidence and saturated incidence, respectively. Its time series evolution is graphically shown in Figure 3, which depicts the dynamics of the sexually active population when the basic reproduction number is greater than unity for the standard and saturated incidence as shown in Figures 3(a) and 3(b), respectively. It is evident from these Figures 3(a) and 3(b) that the disease becomes endemic for both incidence functions.

\section{Dynamics of the model with control measures}

To assess the impact of the three control measures, the model is simulated with public health educational campaigns, condom use, and treatment as the only control measures for a time frame of 50 years (Figure 4). Figure 4(a) shows that there is a steady decrease in the susceptible population due to education and infection. Susceptible individuals join the educated susceptible population, and some are infected resulting into an increase of educated susceptibles before a further decrease due to infection. The populations of aware and unaware infectives decrease due to progression to further stages of infection and treatment of aware infectives. The number of individuals receiving treatment increases when there are more aware infectives and pre-AIDS individuals and decreases when these numbers are low. Figure 4(b) shows that susceptibles, educated susceptibles, unaware infectives, pre-AIDS, and AIDS individuals tend to their steady-state values and aware infectives approach zero. The system settles at an endemic steady-state, and the disease persists in the population, but the infection has been reduced since the reproduction number has been decreased to $R_{e}^{\text {st }}=1.0159$ for the standard incidence and $R_{e}^{\text {sat }}=5.302$ for the saturated incidence. Thus, the control measures introduced are modest and some extra efforts are still needed to stem the tide of the epidemic by bringing the effective reproduction number to less than unity for the standard incidence, but the infection is still very high regardless of the effect of the aforementioned control measures, whence the need for investing in and implementing other control strategies.

\section{Changes in unaware infectives with change in incidence function}

Figure 5(a) shows that the number of unaware infectives decreases almost exponentially over the first 10 years and approaches a steady-state value. Figure 5(b) shows an increase in the measure of the psychological or inhibitory effect, $\omega$, results in a decrease in the number of unaware infectives. 
Table 5: Models parameter values.

\begin{tabular}{lcc}
\hline Parameters & Value & Reference \\
\hline$\Lambda$ & $5000{\mathrm{people} \mathrm{yr}^{-1}}^{-1}$ & Assumed \\
$\mu$ & $0.0196 \mathrm{yr}^{-1}$ & Assumed \\
$\beta$ & $3 \mathrm{yr}^{-1}$ & Assumed \\
$\tau$ & $0.55 \mathrm{yr}^{-1}$ & Assumed \\
$\rho$ & $0.47 \mathrm{yr}^{-1}$ & Assumed \\
$\theta$ & $0.57 \mathrm{yr}^{-1}$ & Assumed \\
$\nu$ & $0.45 \mathrm{yr}^{-1}$ & Assumed \\
$\sigma$ & $0.36 \mathrm{yr}^{-1}$ & Assumed \\
$\eta$ & $0.21 \mathrm{yr}^{-1}$ & Assumed \\
$\alpha$ & $0.22 \mathrm{yr}^{-1}$ & Assumed \\
$\delta$ & $0.33 \mathrm{yr}^{-1}$ & {$[3]$} \\
$r$ & $0.15 \mathrm{yr}^{-1}$ & {$[3]$} \\
$\rho_{1}$ & $0.57 \mathrm{yr}^{-1}$ & Assumed \\
$\rho_{2}$ & $0.32 \mathrm{yr}^{-1}$ & Assumed \\
$\alpha_{1}$ & $0.18 \mathrm{yr}^{-1}$ & Assumed \\
$k_{1}, k_{2}, k_{3}, k_{4}$ & $0.15,0.48,0.0016$ & Assumed \\
$p$ & 0.53 & {$[3]$} \\
$b$ & 0.2 & {$[3]$} \\
$k$ & 0.6 & {$[3]$} \\
$\omega$ & 4.0 & {$[8]$} \\
\hline
\end{tabular}

\section{Prevalence of the disease for the standard incidence}

The prevalence graph, Figure 6(a), increases with a greater gradient for a while and then stabilizes over time, which means that the disease becomes endemic in the population. It is observed in Figure 6(b) that the prevalence curve increases then drops asymptotically but does not approach zero due to reduced number of susceptible individuals over time as most susceptibles join the educated susceptible class and others become affected by the disease. This means that the disease becomes endemic in the population regardless of the effect of the current three interventions in place.

\section{Prevalence of the disease for the saturated incidence}

The numerical simulations for assessing the effects of increasing the saturation rate, $\omega$, are given in Figure 7. An increase in the saturation rate results in a decrease in the prevalence of the infection. The contact rate is decreasing due to increasing the saturation rate but it does not go to zero, implying that the disease becomes endemic in the population despite the impact of the three interventions in place.

\section{Conclusion}

A basic deterministic model of the transmission dynamics of HIV / AIDS was formulated to investigate the role of the incidence function in curtailing an outbreak of the disease. Three control measures including public health educational campaigns, condom use, and treatment were incorporated into the basic model to assess their potential impact on the transmission 


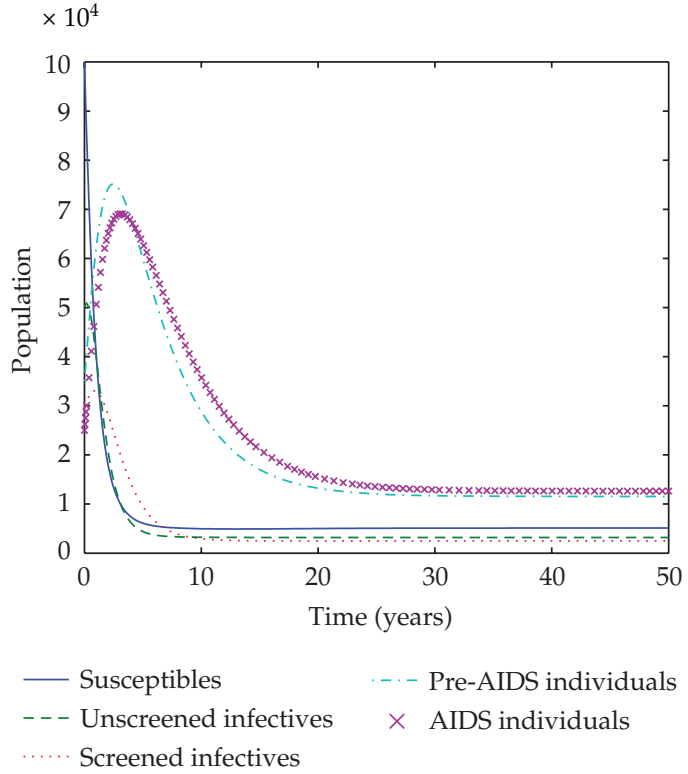

(a)

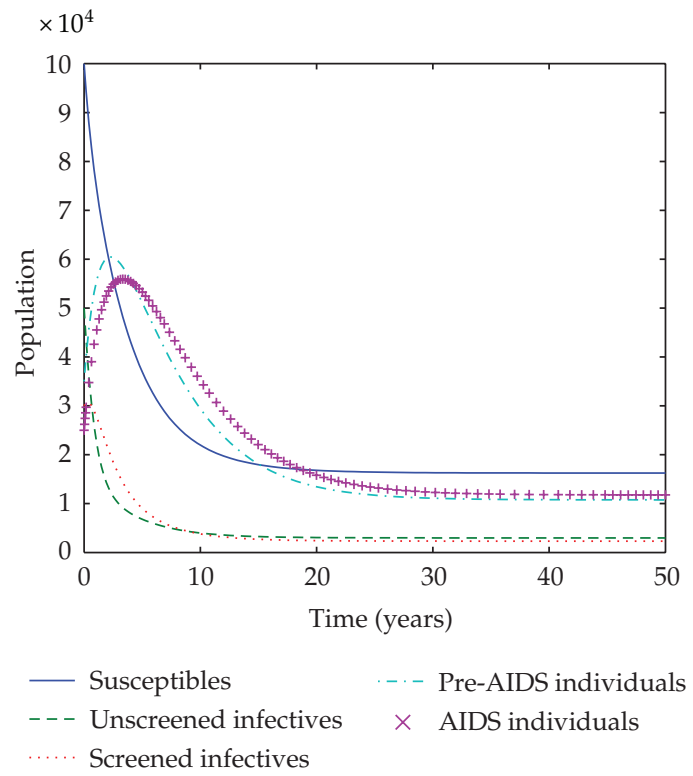

(b)

Figure 3: The basic model with (a) standard incidence and (b) saturated incidence.

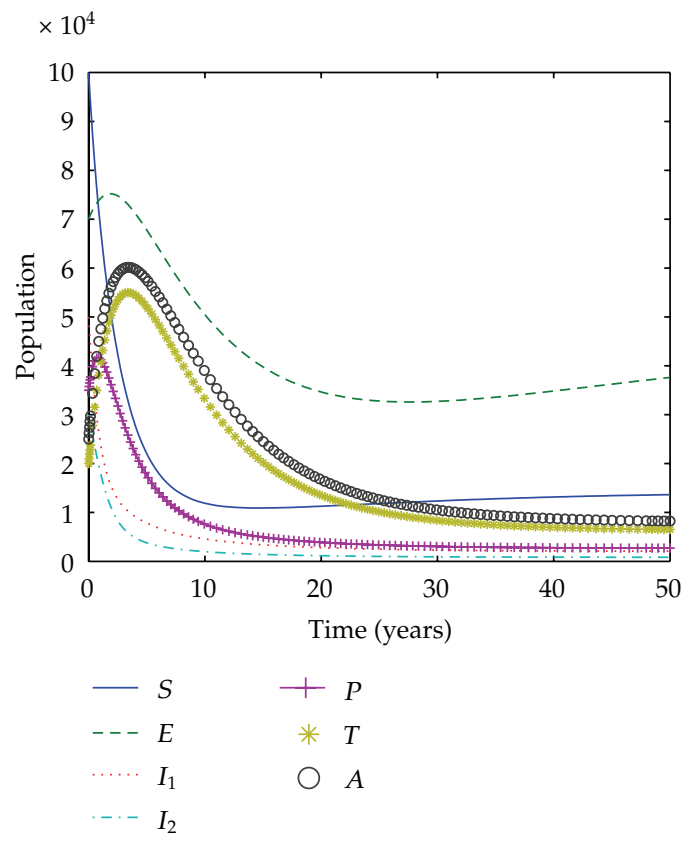

(a)

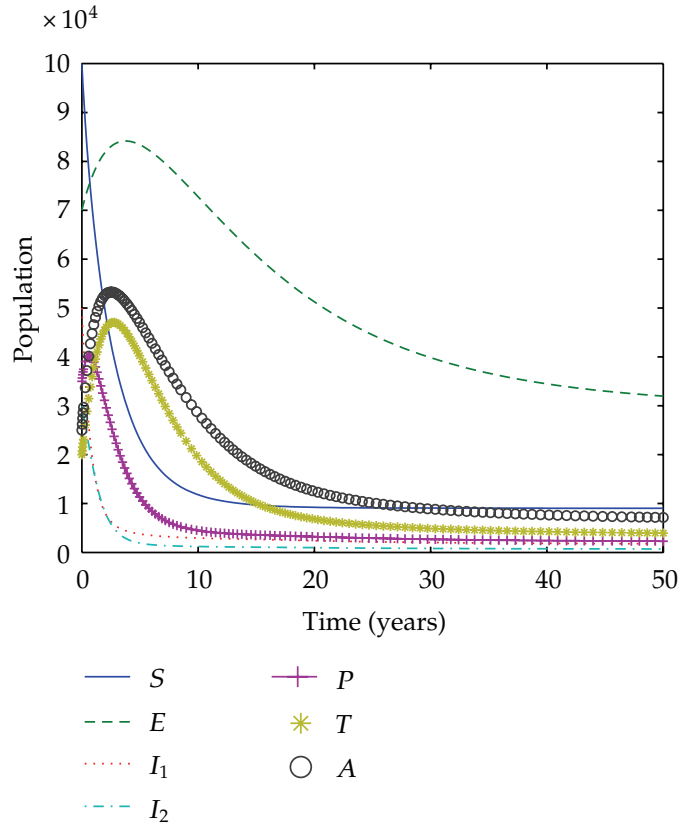

(b)

Figure 4: Population dynamics, when intervention is instituted for 50 years, with (a) standard incidence and (b) saturated incidence. 


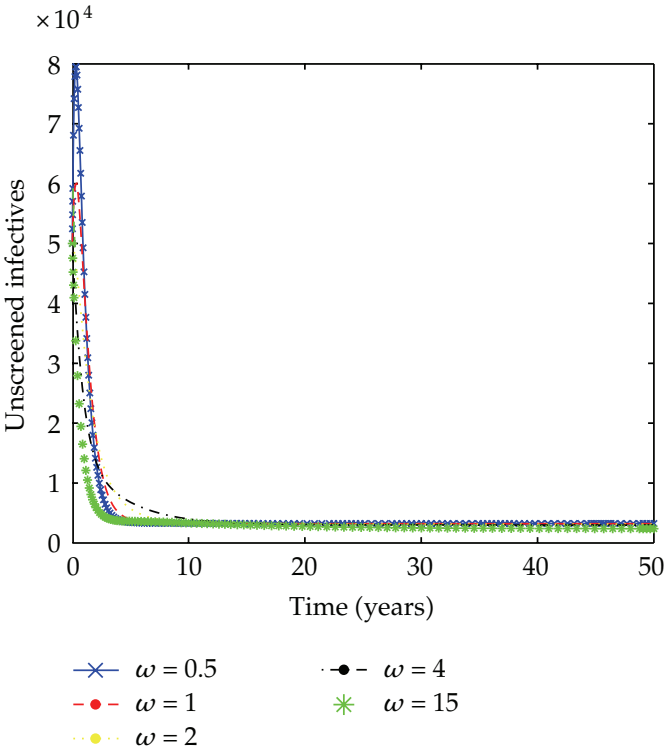

(a)

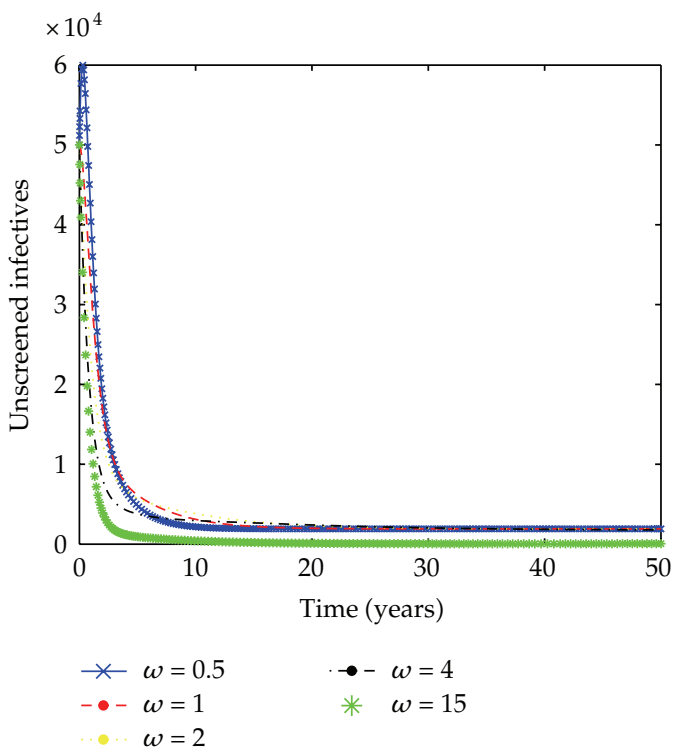

(b)

Figure 5: Change of unaware infectives with (a) standard incidence and saturated incidence for the basic model and (b) standard incidence and saturated incidence for the intervention model.

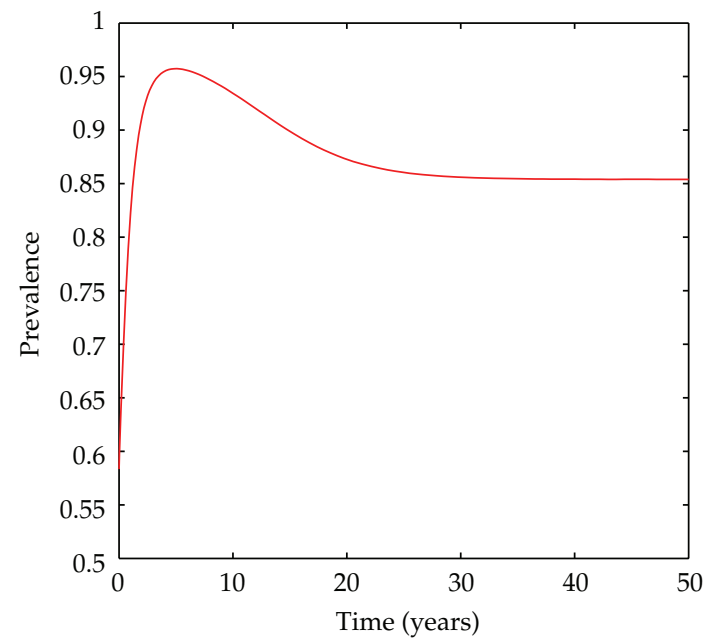

(a)

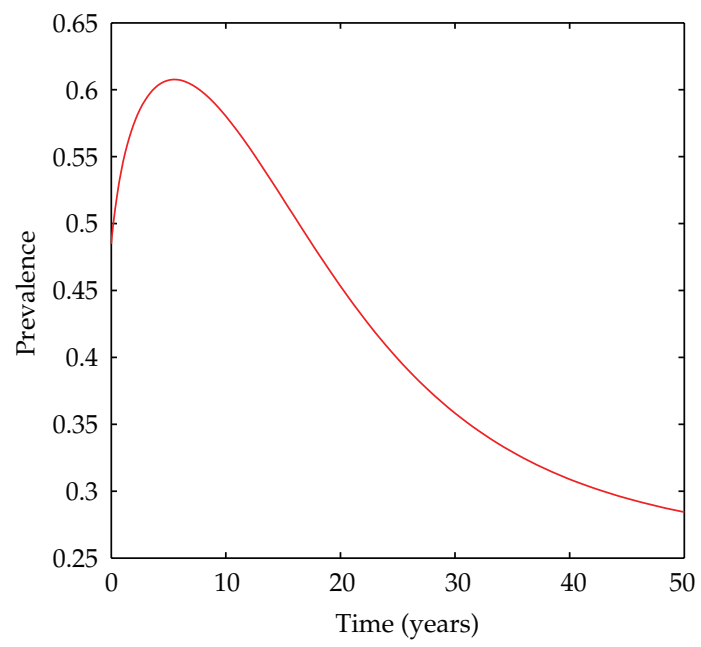

(b)

Figure 6: Prevalence of the disease for the (a) basic and (b) intervention models with standard incidence.

dynamics of the disease with change of incidence function. The qualitative features of the models were investigated.

The model has two equilibria, the disease-free equilibrium and the endemic equilibrium. Theoretical results show that the disease-free equilibrium is stable (locally and globally) when $R_{0}^{\text {st }} \leq 1$ and $R_{0}^{\text {sat }} \leq 1$ for standard incidence and saturated incidence, respectively. If both $R_{0}^{\text {st }}$ and $R_{0}^{\text {sat }}$ are greater than unity, then the disease will persist in 


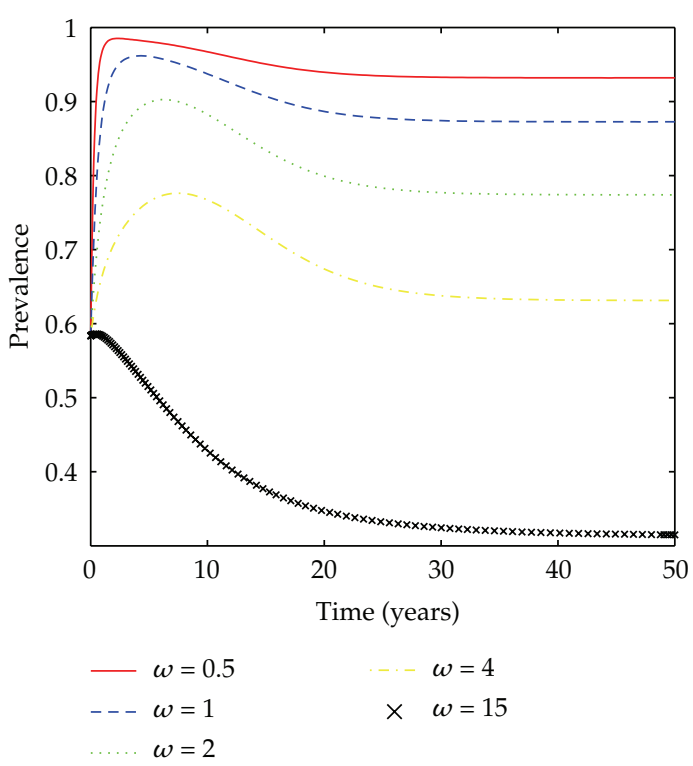

(a)

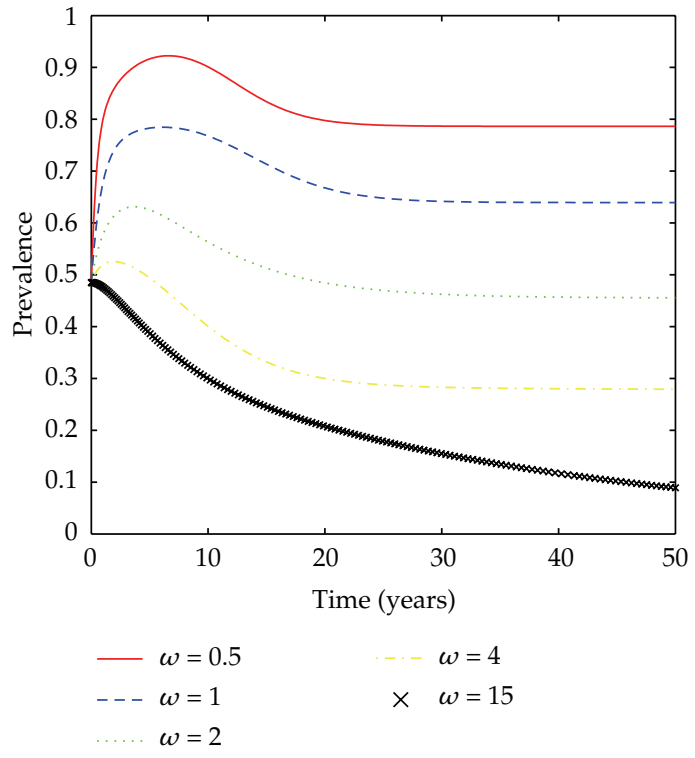

(b)

Figure 7: Prevalence of the disease for the (a) basic and (b) intervention models with saturated incidence.

the population. In this case, a unique endemic equilibrium exists, is locally and globally asymptotically stable for both models. The condition under which the disease persists in the population with saturated incidence is also derived. It was also shown that the basic reproduction number for the saturated incidence model is greater than that for the standard incidence model, that is, $R_{0}^{\text {sat }}>R_{0}^{\text {st }}$. The local stability of the endemic equilibrium point was proved for the standard incidence model using the center manifold theory. The models did not exhibit the phenomenon of backward bifurcation where a stable disease-free equilibrium coexists with a stable endemic equilibrium for a certain range of associated reproduction number less than unity for both incidence functions. Consequently, both the disease-free and endemic equilibria for these models are stable (both locally and globally) when $R_{e}^{\text {st }}, R_{e}^{\text {sat }} \leq 1$ and when $R_{e}^{\text {st }}, R_{e}^{\text {sat }}>1$, respectively. Note that $R_{e}^{\text {sat }}>R_{e}^{\text {st }}$.

Numerical simulations were obtained using some demographic data from the literature and the remaining parameter values assumed for the purpose of illustration. The saturation impact is measured by the parameter $\omega$. Though the basic as well as the effective reproduction numbers, $R_{0}^{\text {sat }}$ and $R_{e}^{\text {sat }}$, do not depend on $\omega$ explicitly, numerical simulations indicate that the number of infectives decreases as $\omega$ increases (Figure 7). The larger the value of $\omega$ is, the smaller the inhibitory effect is, and vice-versa. An increase in $\omega$ leads to a reduction in the prevalence of the disease.

Our results highlight the potential impact the incidence functions may play on the behavioral dynamics of the model and underscore the need for further work on the incidence functions used in HIV models. The number of HIV-infected individuals has slowed considerably following a rapid and aggressive information campaign and implementation of various control strategies in recent years. Prevalence has also fallen after peaking in the mid-1990s; consequently, a saturation incidence model currently seems to make more epidemiological sense than the standard incidence for modeling the current HIV/AIDS epidemic. However, caution should be paid in choosing and interpreting results as the choice 
of the incidence function can greatly influence the results by either over or underestimating the measure of initial disease spread. Whenever data is available, fitting the models to data, even though different incidence functions would probably lead to different parameter values can as well provide some information given experts opinion on the potential true course of the epidemic on which form of the incidence function to choose. This is a daunting task for most epidemic, especially given that, more often, data are not readily available and hard to come by.

\section{Acknowledgment}

E. L. Kateme acknowledges with thanks the support in part of the the Norad's programme for Master Studies (in Mathematical Modelling) at the University of Dar es Salaam.

\section{References}

[1] N. Hussaini, M. Winter, and A. B. Gumel, "Qualitative assessment of the role of public health education program on HIV transmission dynamics," Mathematical Medicine and Biology, vol. 28, no. 3, pp. 245-270, 2011.

[2] Z. Mukandavire and W. Garira, "Effects of public health educational campaigns and the role of sex workers on the spread of HIV / AIDS among heterosexuals," Theoretical Population Biology, vol. 72, no. 3, pp. 346-365, 2007.

[3] Z. Mukandavire, W. Garira, and J. M. Tchuenche, "Modelling effects of public health educational campaigns on HIV / AIDS transmission dynamics," Applied Mathematical Modelling, vol. 33, no. 4, pp. 2084-2095, 2009.

[4] J. Musgrave and J. Watmough, "Examination of a simple model of condom usage and individual withdrawal for the HIV epidemic," Mathematical Biosciences and Engineering, vol. 6, no. 2, pp. 363-376, 2009.

[5] O. Sharomi, C. N. Podder, A. B. Gumel, E. H. Elbasha, and J. Watmough, "Role of incidence function in vaccine-induced backward bifurcation in some HIV models," Mathematical Biosciences, vol. 210, no. 2, pp. 436-463, 2007.

[6] F. Nyabadza, C. Chiyaka, Z. Mukandavire, and S. D. Hove-Musekwa, "Analysis of an HIV/AIDS model with public-health information campaigns and individual withdrawal," Journal of Biological Systems, vol. 18, no. 2, pp. 357-375, 2010.

[7] J. Zhang and Z. Ma, "Global dynamics of an SEIR epidemic model with saturating contact rate," Mathematical Biosciences, vol. 185, no. 1, pp. 15-32, 2003.

[8] D. Xiao and S. Ruan, "Global analysis of an epidemic model with nonmonotone incidence rate," Mathematical Biosciences, vol. 208, no. 2, pp. 419-429, 2007.

[9] W. Liu, H. W. Hethcote, and S. A. Levin, "Dynamical behavior of epidemiological models with nonlinear incidence rates," Journal of Mathematical Biology, vol. 25, pp. 359-380, 1989.

[10] V. Capasso and G. Serio, "A generalization of the Kermack-McKendrick deterministic epidemic model," Mathematical Biosciences, vol. 42, no. 1-2, pp. 43-62, 1978.

[11] E. Mtisi, H. Rwezaura, and J. M. Tchuenche, "A mathematical analysis of malaria and tuberculosis co-dynamics," Discrete and Continuous Dynamical Systems B, vol. 12, no. 4, pp. 827-864, 2009.

[12] H. W. Hethcothe, "The mathematics of infectious disease," SIAM Review, vol. 42, pp. 599-653, 2000.

[13] P. van den Driessche and J. Watmough, "Reproduction numbers and sub-threshold endemic equilibria for compartmental models of disease transmission," Mathematical Biosciences, vol. 180, pp. 29-48, 2002.

[14] J. Carr, Applications of Centre Manifold Theory, vol. 35, Springer, New York, NY, USA, 1981.

[15] C. Castillo-Chavez and B. Song, "Dynamical models of tuberculosis and their applications," Mathematical Biosciences and Engineering, vol. 1, pp. 361-404, 2004.

[16] O. Diekmann, J. A. P. Heesterbeek, and J. A. J. Metz, “On the definition and the computation of the basic reproduction ratio $R_{0}$ in models for infectious diseases in heterogeneous populations," Journal of Mathematical Biology, vol. 28, no. 4, pp. 365-382, 1990. 


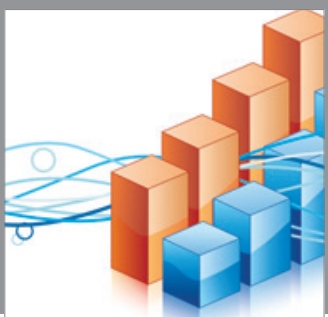

Advances in

Operations Research

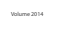

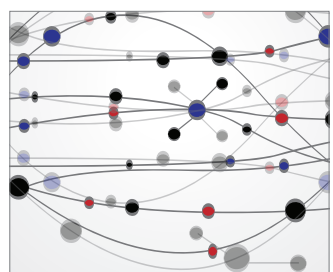

\section{The Scientific} World Journal
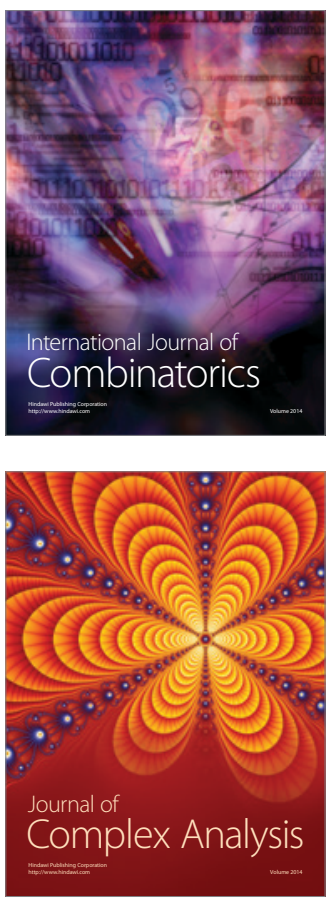

International Journal of

Mathematics and

Mathematical

Sciences
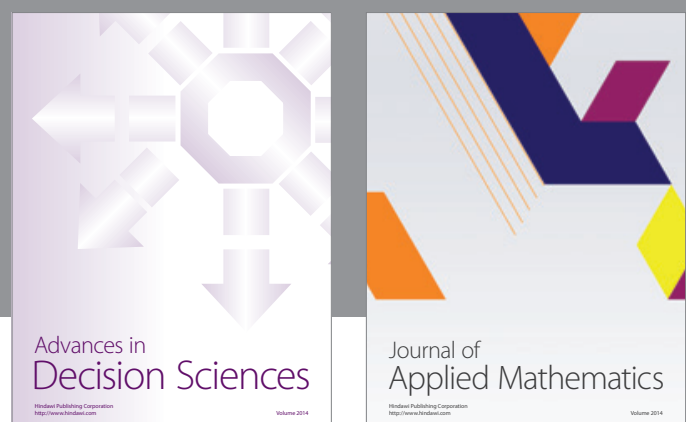

Journal of

Applied Mathematics
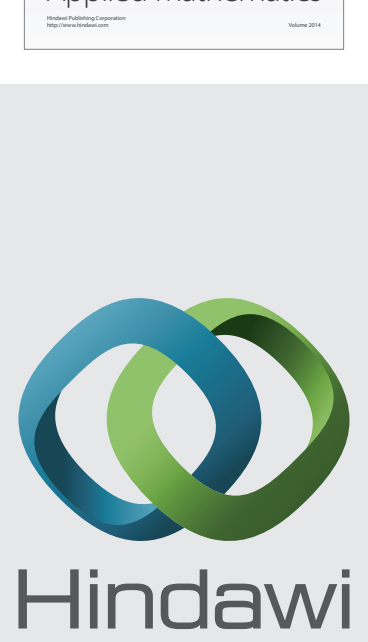

Submit your manuscripts at http://www.hindawi.com
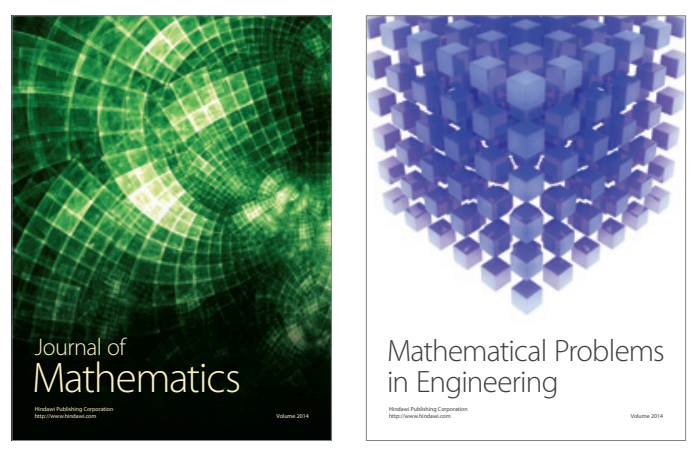

Mathematical Problems in Engineering
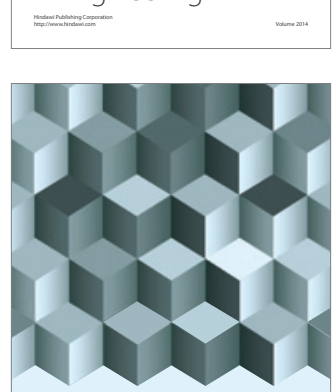

Journal of

Function Spaces
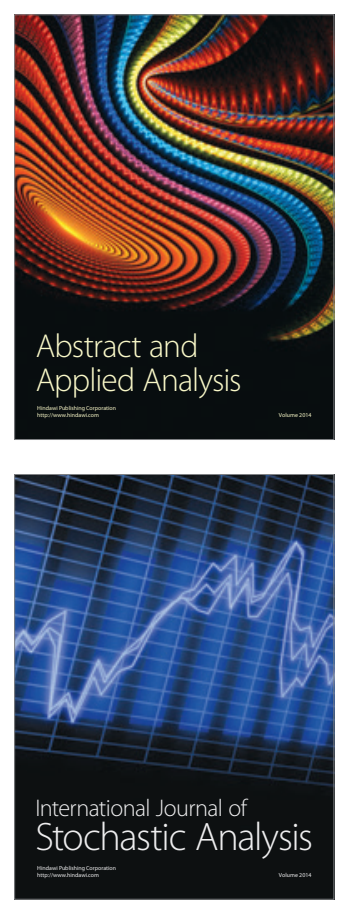

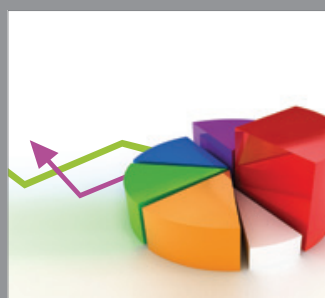

ournal of

Probability and Statistics

Promensencen
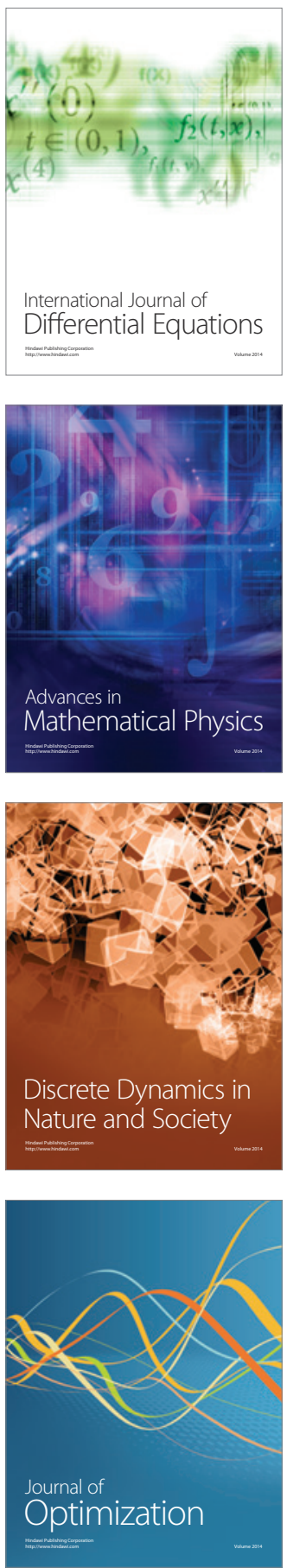